\title{
Ultrafiltration in patients with decompensated heart failure and diuretic resistance: an Asian centre's experience
}

Loon Yee Louis $\underline{T e O}^{1}$, MBBS, MRCP, Choon Pin $\underline{\operatorname{Lim}}^{1}$, MBBS, MRCP, Chia Lee $\underline{\mathrm{NeO}}^{1}, \mathrm{BSN}$, Lee Wah $\underline{\mathrm{TeO}}^{1}, \mathrm{BSN}$, Swee Ling Elaine $\underline{\mathrm{Ng}}^{1}$, BSN, Laura Lihua $\underline{\text { Chan }}^{1}$, MBBS, MRCP, Manish Kaushik ${ }^{2}$, MBBS, MRCP,

Kheng Leng David $\underline{\operatorname{Sim}}^{1}$, MBBS, MRCP

INTRODUCTION Diuretics are the mainstay of therapy for restoring the euvolaemic state in patients with decompensated heart failure. However, diuretic resistance remains a challenge.

METHODS We conducted a retrospective cohort study to examine the efficacy and safety of ultrafiltration (UF) in 44 hospitalised patients who had decompensated heart failure and diuretic resistance between October 2011 and July 2013. RESULTS Among the 44 patients, 18 received UF (i.e. UF group), while 26 received diuretics (i.e. standard care group). After 48 hours, the UF group achieved lower urine output $(1,355 \mathrm{~mL}$ vs. $3,815 \mathrm{~mL}, \mathrm{p}=0.0003)$, greater fluid loss $(5,058 \mathrm{~mL}$ vs. $1,915 \mathrm{~mL}, \mathrm{p}<0.0001)$ and greater weight loss $(5.0 \mathrm{~kg}$ vs. $1.0 \mathrm{~kg}, \mathrm{p}<0.0001)$ than the standard care group. The UF group also had a shorter duration of hospitalisation ( 5.0 days vs. 9.5 days, $p=0.0010$ ). There were no differences in the incidence of 30-day emergency department visits and rehospitalisations for heart failure between the two groups. At 90 days, the UF group had fewer emergency department visits $(0.2 \mathrm{vs} .0 .8, p=0.0500)$ and fewer rehospitalisations for heart failure ( 0.3 vs. $1.0, p=0.0442$ ). Reduction in $E Q-5 D^{\text {TM }}$ scores was greater in the UF group, both at discharge ( 2.7 vs. $1.4, p=0.0283)$ and 30 days $(2.5$ vs. $0.3, p=0.0033)$. No adverse events were reported with UF.

CONCLUSION UF is an effective and safe treatment that can improve the health outcomes of Asian patients with decompensated heart failure and diuretic resistance.

Keywords: Asia, diuretic resistance, efficacy and safety, heart failure, ultrafiltration

\section{INTRODUCTION}

A high prevalence of heart failure (HF) results in a significant burden to society in terms of morbidity and mortality, both internationally ${ }^{(1,2)}$ and locally..$^{(3,4)}$ Diuretics are the only drugs that can adequately control the fluid retention that occurs with HF; thus, they are the most effective group of drugs for providing rapid symptomatic benefits. ${ }^{(5)}$ Despite treatment with intravenous diuretics, a large proportion of hospitalised HF patients fail to achieve adequate diuresis and weight loss, and have unresolved symptoms and high readmission rates. ${ }^{(6)}$ Furthermore, patients with renal impairment and diuretic resistance have been reported to have a prolonged hospital stay. ${ }^{(7,8)}$

Although to date, there is no consensus on the definition of diuretic resistance, it is often described when there is persistent clinical congestion due to inadequate diuresis and natriuresis after administration of at least $80 \mathrm{mg}$ of furosemide daily or an equivalent dose of diuretics. ${ }^{(9-12)}$ The prevalence of diuretic resistance among patients with HF is unknown. ${ }^{(10)}$ The concept of extracorporeal removal of fluid using ultrafiltration (UF) has been reported for over 50 years. ${ }^{(13)}$ Its use in HF patients was initially reported in publications in the mid-1980s. ${ }^{(14,15)}$ In the past, the use of UF was limited by the need for a large, cumbersome haemodialysis machine and the use of a large central venous line at high flow rate for blood removal. The availability of new UF devices, such as the Aquadex FlexFlow ${ }^{\mathrm{TM}}$ Fluid Removal
System (Gambro, Deerfield, IL, USA), which is much smaller, more portable and requires only a peripheral venous catheter, overcomes the aforementioned limitations and elevates the potential of UF in HF treatment. ${ }^{(16)}$ UF has been proven to be a safe and effective alternative to intravenous diuretics in the treatment of decompensated HF. ${ }^{(17,18)}$ However, there have not been any clinical trials assessing the use of UF in decompensated HF patients with diuretic resistance. The present study aimed to determine the efficacy and safety of performing UF in patients with decompensated $\mathrm{HF}$ and diuretic resistance, by evaluating the use of UF in a cohort of patients who were hospitalised in our centre.

\section{METHODS}

From October 2011 to July 2013, a total of 1,464 patients admitted to the National Heart Centre Singapore, Singapore, with a diagnosis of decompensated HF were screened for eligibility to undergo UF. 44 consecutive patients who remained fluid overloaded despite being treated with diuretic therapy were offered UF therapy. Among these 44 patients, 18 received UF (i.e. UF group), while 26 declined UF and continued to receive treatment with diuretics. The latter (i.e. standard care group) served as controls in this study. We performed a retrospective cohort study by examining the medical records of the aforementioned 44 patients, to assess the efficacy and safety of UF in patients with decompensated HF and diuretic resistance.

${ }^{1}$ Department of Cardiology, National Heart Centre Singapore, ${ }^{2}$ Department of Renal Medicine, Singapore General Hospital, Singapore 
To be eligible for UF, the patients must fulfil the following criteria: (a) aged > 21 years; (b) able to provide informed consent; (c) hospitalised with a primary diagnosis of acute decompensated HF; (d) diuretic resistant; and (e) hypervolaemic. Diuretic resistance was defined as having a urine output $<125 \mathrm{~mL} / \mathrm{hr}$ after the administration of at least $80 \mathrm{mg}$ of intravenous frusemide per day. The patient was deemed to be hypervolaemic if at least two of the following characteristics were present: (a) peripheral oedema $\geq 2+$; (b) jugular venous distension; (c) radiographic pulmonary oedema or pleural effusion; (d) enlarged liver or ascites; (e) pulmonary rales, paroxysmal nocturnal dyspnoea or orthopnoea; (f) S3 gallop; and $(\mathrm{g})$ rapid weight gain of $2 \mathrm{~kg}$ over dry weight. The exclusion criteria for UF were: (a) acute coronary syndrome; (b) end-stage renal disease requiring dialysis; (c) Stage 4 and above chronic kidney disease (calculated glomerular filtration rate $\leq 30 \mathrm{~mL} / \mathrm{min}$ ); (d) requires intravenous inotropes or vasoactive drugs; (e) systolic blood pressure < $85 \mathrm{mmHg}$; (f) haematocrit $>45 \%$; (g) heparin allergy or contraindication to the use of anticoagulation; (h) sepsis or systemic infection; (i) pregnancy; (j) co-existing diseases that are expected to result in death within three months or prolonged hospitalisation; ( $k$ ) use of radiocontrast material in the last 72 hours; or (I) inability to obtain venous access.

The Aquadex FlexFlow Fluid Removal System was used for all patients who received UF. For venous access, a 6-Fr, central, dual-lumen catheter was placed in the internal jugular vein or the femoral vein of the patients. The patients then received a single course of UF; the fluid removal rate (up to a maximum of $500 \mathrm{~mL} / \mathrm{hr}$ ) was left to the discretion of the treating HF specialist. UF was performed in the general ward and operated by an HF nurse (who was specially trained in UF management) under the guidance of the treating HF specialist. UF was allowed to run until the patients achieved euvolaemia or the system clotted prematurely before euvolaemia was attained. Oral and intravenous diuretics were discontinued during UF. Intravenous heparin was given to the UF group according to the standard protocol in order to maintain a partial thromboplastin time of $60-80$ seconds.

Patients who were not eligible for or refused treatment with UF were treated with intravenous diuretics, which is the standard of care. The doses of diuretics were titrated by the treating physician to manage the signs and symptoms of congestion. The use of intravenous inotropes and vasodilators was also determined by the treating physician based on the patient's urine output, blood pressure and renal function.

The clinical outcomes of interest included net fluid and weight loss at 48 hours after treatment. The 48 -hour net fluid loss was calculated by measuring the patient's total fluid intake and output on treatment assignment and at 48 hours. The 48-hour weight loss was the difference between the patient's weight on treatment and at 48 hours. Subjective assessments of the patient's health outcomes at discharge and 30 days were evaluated using the standardised EQ-5D-3LTM health questionnaire. ${ }^{(19)}$ This questionnaire consists of the following five dimensions: mobility; self-care; usual activities; pain/discomfort; and anxiety/depression. Each dimension is measured on a scale of $1-3$, where 1 indicates the absence of problems, 2 indicates the presence of some problems and 3 indicates the presence of extreme problems. The duration of hospitalisation, the number of emergency department visits and the number of rehospitalisations for HF within 30 days and 90 days were retrieved from the electronic medical records. The safety endpoints of UF were assessed by examining the incidence of hypotension, bleeding, electrolyte imbalance, worsening renal function and line-related complications. We compared the blood pressure, and haemoglobin, serum sodium, potassium and creatinine levels of the patients before and after UF was performed.

Categorical variables were expressed as frequencies and percentages, and compared using Fisher's exact test. Normally distributed continuous variables were presented as mean \pm standard deviation and compared using two-tailed, unpaired Student's $t$-test. Continuous variables that were not normally distributed were presented as median (interquartile range $[I Q R])$, and compared using Wilcoxon rank-sum test. A p-value $\leq 0.05$ was considered statistically significant.

\section{RESULTS}

Among the 1,464 patients admitted to our centre with decompensated HF, 44 (3.0\%) who were identified as being diuretic-resistant made up the study cohort. The median age of the study cohort was 63.2 (IQR 56.5-70.5) years and $84.1 \%$ of the patients were male. The patients were of the following ethnicities: Chinese ( $n=28)$; Malay $(n=9)$; and Indian $(n=7)$. More than half of the patients $(63.6 \%)$ had prior admissions due to HF in the previous 12 months. The majority were classified as New York Heart Association (NYHA) functional class III (75.0\%) and only a small number of patients were in NYHA IV (11.4\%). The median ejection fraction was $29.0 \%$. In terms of the medications that the patients were receiving prior to the index hospitalisation, $75.0 \%$ were on beta blockers, $63.6 \%$ on either angiotensinconverting enzyme inhibitors or angiotensin receptor blockers, and $47.7 \%$ on mineralocorticoid receptor antagonists. Most of the patients (93.2\%) were also on oral frusemide (median dose of $120 \mathrm{mg} /$ day) prior to the index hospitalisation. The median serum creatinine level of the study cohort was $126 \mu \mathrm{mol} / \mathrm{L}$. The baseline demographic and clinical characteristics of the patients were similar between the UF group $(n=18)$ and the standard care group $(\mathrm{n}=26)$ (Table $\mathrm{I})$.

For each patient in the UF group, a single session of UF was performed at an average fluid removal rate of $314 \pm 99 \mathrm{~mL} / \mathrm{hr}$. The mean duration of UF was $18.4 \pm 13.2$ hours, and the mean fluid volume removed was 5,836 $\pm 3,800 \mathrm{~mL}$. The standard care group received a mean daily dose of intravenous frusemide $167 \pm 57 \mathrm{mg}$. In the standard care group, intravenous inotropes were used in five patients and intravenous vasodilator was used in one patient; none of the patients in the UF group required the use of inotropes or vasodilators during their subsequent HF treatment.

At 48 hours, the UF group had a significantly lower median urine output than the standard care group (1,355 [IQR 1,045-2,485] mL vs. 3,815 [IQR 2,186-4,620] mL, $\mathrm{p}=0.0003$ ). However, the UF group also had a greater median net fluid loss than the standard care group at 48 hours $(5,058$ [IQR 3,959-8,107] 
Table I. Baseline demographics and clinical characteristics of the patients with heart failure (HF), according to the treatment received ( $=44)$.

\begin{tabular}{|c|c|c|c|}
\hline \multirow[t]{2}{*}{ Characteristic } & \multicolumn{2}{|c|}{$\%$} & \multirow{2}{*}{ p-value } \\
\hline & UF group $(n=18)$ & Standard care group $(n=26)$ & \\
\hline Age* (yr) $^{*}$ & $61(55-64)$ & $64(58-72)$ & 0.201 \\
\hline Male gender & 94.4 & 76.9 & 0.211 \\
\hline \multicolumn{4}{|l|}{ Ethnicity } \\
\hline Chinese & 61.1 & 65.4 & 1.002 \\
\hline Malay & 16.7 & 23.1 & 0.716 \\
\hline Indian & 22.2 & 11.5 & 0.419 \\
\hline \multicolumn{4}{|l|}{ Comorbidity } \\
\hline Prior myocardial infarction & 72.2 & 57.7 & 0.361 \\
\hline Diabetes mellitus & 72.2 & 73.1 & 1.030 \\
\hline Hypertension & 66.7 & 65.4 & 1.025 \\
\hline History of atrial fibrillation & 22.2 & 46.1 & 0.125 \\
\hline Prior admission for $\mathrm{HF}$ in $\leq 12 \mathrm{mth}$ & 55.6 & 69.2 & 0.525 \\
\hline No. of hospitalisations for HF in $\leq 12 \mathrm{mth}^{*}$ & $1(0-3)$ & $1(0-3)$ & 0.535 \\
\hline Left ventricular ejection fraction* $(\%)$ & $29(20-46)$ & $24(20-30)$ & 0.112 \\
\hline \multicolumn{4}{|l|}{ NYHA functional class } \\
\hline III & 61.1 & 84.6 & 0.093 \\
\hline IV & 16.7 & 7.7 & 0.386 \\
\hline \multicolumn{4}{|l|}{ Vital signs* } \\
\hline Systolic blood pressure (mmHg) & $106(102-135)$ & $110(97-123)$ & 0.370 \\
\hline Heart rate (beats/min) & $70(63-80)$ & $81(67-89)$ & 0.081 \\
\hline Weight (kg) & $66(58-74)$ & $67(57-85)$ & 0.712 \\
\hline \multicolumn{4}{|l|}{ Clinical signs of HF on admission } \\
\hline Pulmonary crepitations & 88.9 & 88.4 & 1.023 \\
\hline Jugular venous distension & 88.9 & 80.7 & 0.682 \\
\hline Peripheral oedema & 94.4 & 100.0 & 0.409 \\
\hline \multicolumn{4}{|l|}{ Laboratory measurements* } \\
\hline Serum urea $(\mathrm{mmol} / \mathrm{L})$ & $9.6(7.3-14.6)$ & $9.7(7.3-11.8)$ & 0.551 \\
\hline Serum creatinine $(\mu \mathrm{mol} / \mathrm{L})$ & $124(98-154)$ & $127(108-160)$ & 0.567 \\
\hline Serum sodium (mmol/L) & $136(134-140)$ & $137(135-139)$ & 0.810 \\
\hline Serum potassium (mmol/L) & $4.1(4-4.3)$ & $4.2(3.8-4.5)$ & 0.368 \\
\hline Haemoglobin $(\mathrm{g} / \mathrm{dL})$ & $11.6(10.1-13.4)$ & $12.7(11.1-14.4)$ & 0.189 \\
\hline Haematocrit (\%) & $36.7(30.4-41.3)$ & $29.3(33.2-43.5)$ & 0.267 \\
\hline NT-proBNP (pg/mL) & $6,695(3,213-9,814)$ & $6,252(3,191-13,258)$ & 0.645 \\
\hline \multicolumn{4}{|c|}{ Medication received before index hospitalisation } \\
\hline Beta blocker & 72.2 & 76.9 & 0.738 \\
\hline ACE inhibitor & 38.9 & 61.5 & 0.220 \\
\hline Angiotensin receptor blocker & 11.1 & 11.5 & 1.002 \\
\hline Minerolocorticoid receptor antagonist & 55.6 & 42.3 & 0.541 \\
\hline Calcium channel blocker & 5.6 & 11.5 & 0.634 \\
\hline Digoxin & 33.3 & 26.9 & 0.742 \\
\hline Frusemide & 88.9 & 96.2 & 0.558 \\
\hline Frusemide dose ${ }^{*}$ (mg/day) & $120(75-160)$ & $120(55-120)$ & 0.205 \\
\hline
\end{tabular}

*Data presented as median (interquartile range). ACE: angiotensin-converting enzyme; NT-proBNP: N-terminal of the prohormone brain natriuretic peptide; NYHA: New York Heart Association; UF: ultrafiltration

$\mathrm{mL}$ vs. 1,915 [IQR 832-2,694] $\mathrm{mL}, \mathrm{p}<0.0001)$. The median weight loss at 48 hours was significantly greater in the UF group than in the standard care group (5.0 [IQR 2.9-8.7] kg vs. 1.0 [IQR 0.3-3.1] kg, p < 0.0001).

Importantly, the UF group had a shorter median length of stay for the index hospitalisation than the standard care group (5.0 [IQR 3.8-7.3] days vs. 9.5 [IQR 6.8-11.0] days, $\mathrm{p}=0.0010$ ). Within
30 days, there were no statistically significant differences in the number of emergency department visits and rehospitalisations for HF between the two groups. The mean number of 30-day emergency department visits for HF in the UF group was $0.1 \pm 0.3$, while that for the standard care group was $0.4 \pm 0.6(p=0.1203)$. The mean number of 30-day rehospitalisations for $\mathrm{HF}$ in the UF group was $0.2 \pm 0.4$, while that for the standard care group was 
$0.5 \pm 0.6(p=0.2238)$. Within 90 days, there was a significantly lower number of emergency department visits for HF in the UF group than in the standard care group $(0.2 \pm 0.4$ vs. $0.8 \pm 1.3$, $p=0.0500$ ). There was also a decrease in the number of 90-day rehospitalisations for HF in the UF group as compared to the standard care group $(0.3 \pm 0.7$ vs. $1.0 \pm 1.2, p=0.0442)$. The use of UF was associated with an improvement in the patient's health outcomes. This was reflected in the greater reduction of EQ-5D scores in the UF group as compared to the standard care group at discharge $(2.7 \pm 1.4$ vs. $1.4 \pm 2.4, \mathrm{p}=0.0283)$ and 30 days $(2.5 \pm 1.5$ vs. $0.3 \pm 2.8, p=0.0033)$. The clinical outcomes of the two groups are summarised in Table II.

When we compared the blood pressure, and haemoglobin, serum sodium, potassium and creatinine levels of the patients who underwent UF, before and after UF was performed, no clinically significant changes were found (Table III). There were no linerelated complications reported in the UF group.

\section{DISCUSSION}

Previous trials have established UF as a safe and effective treatment modality for patients with decompensated HF. ${ }^{(17,18)}$ The American College of Cardiology Foundation/American Heart Association Task Force (ACCF/AHA) 2013 guidelines on HF recommend UF as a Class IIb (Level of evidence: B) treatment strategy for: (a) patients with obvious volume overload, to alleviate congestive symptoms and fluid weight; and (b) patients with refractory congestion who are not responding to medical therapy. ${ }^{(5)}$ On the other hand, the European Society of Cardiology (ESC) 2012 guidelines for HF do not give specific class recommendations for UF but suggest the use of UF in patients who are resistant to diuretics. ${ }^{(20)}$ These recommendations are based primarily on the 2007 study, Ultrafiltration versus Intravenous Diuretics for Patients Hospitalized for Acute Decompensated Congestive Heart Failure (UNLOAD), which is the largest randomised trial comparing UF against intravenous diuretic therapy in hypervolaemic HF patients. ${ }^{(18)}$ Despite these recommendations, UF via the portable Aquadex FlexFlow system in decompensated HF patients with diuretic resistance has not been validated. There is a previous study that showed that slow continuous UF via a haemodialysis machine resulted in poor clinical outcomes among refractory patients with advanced decompensated HF. ${ }^{(21)}$

The present study is a single centre's clinical experience of a cohort of patients hospitalised with HF and diuretic resistance, undergoing UF; the outcomes of this cohort of patients were compared to those of another cohort of patients who were eligible for UF but opted to undergo standard diuretic therapy. We demonstrated significantly greater fluid and weight loss with UF than intravenous diuretics, despite a significantly lower urine output in the UF group. These results, which are comparable to those of previous studies, ${ }^{(16,17,19)}$ are not unexpected, as the fluid removal rate can be adjusted using the UF device. Conversely, the urine output in the standard care group was variable and mostly non-substantial (especially since the standard care group was also made up of patients with diuretic resistance).

The effectiveness of UF in removing fluid translated into improved clinical outcomes, shorter hospital stays, as well as reductions in the number of emergency department visits and readmissions for HF. Our data indicated that the UF group had

Table II. Clinical outcomes of the patients with heart failure (HF), according to the treatment received $(\mathrm{n}=44)$.

\begin{tabular}{|c|c|c|c|}
\hline \multirow[t]{2}{*}{ Clinical outcome } & \multicolumn{2}{|c|}{ Mean \pm standard deviation } & \multirow[t]{2}{*}{ p-value } \\
\hline & UF group $(n=18)$ & Standard care group $(n=26)$ & \\
\hline 48-hr net fluid loss* $(\mathrm{mL})$ & $5,058(3,959-8,107)$ & $1,915(832-2,694)$ & $<0.0001$ \\
\hline 48-hr weight loss* (kg) & $5.0(2.9-8.7)$ & $1.0(0.3-3.1)$ & $<0.0001$ \\
\hline Duration of index hospitalisation* (day) & $5.0(3.8-7.3)$ & $9.5(6.8-11.0)$ & 0.0010 \\
\hline No. of ED visits for HF within 30 days & $0.1 \pm 0.3$ & $0.4 \pm 0.6$ & 0.1203 \\
\hline No. of ED visits for HF within 90 days & $0.2 \pm 0.4$ & $0.8 \pm 1.3$ & 0.0500 \\
\hline No. of readmissions for HF within 90 days & $0.3 \pm 0.7$ & $1.0 \pm 1.2$ & 0.0442 \\
\hline Reduction in EQ-5D score at discharge & $2.7 \pm 1.4$ & $1.4 \pm 2.4$ & 0.0283 \\
\hline Reduction in EQ-5D score at 30 days & $2.5 \pm 1.5$ & $0.3 \pm 2.8$ & 0.0033 \\
\hline
\end{tabular}

*Data presented as median (interquartile range). ED: emergency department; UF: ultrafiltration

Table III. Safety endpoints of ultrafiltration (UF) $(n=18)$.

\begin{tabular}{lcc}
\hline Safety endpoint & \multicolumn{1}{c}{ Median (interquartile range) } & p-value \\
\cline { 2 - 3 } & Befter UF & $108(98-124)$ \\
\hline Systolic blood pressure $(\mathrm{mmHg})$ & $106(102-135)$ & $62(60-70)$ \\
Diastolic blood pressure $(\mathrm{mmHg})$ & $74(59-81)$ & 0.334 \\
Haemoglobin $(\mathrm{g} / \mathrm{dL})$ & $11.6(10.1-13.4)$ & $11.7(9.6-13.0)$ \\
Serum sodium $(\mathrm{mmol} / \mathrm{L})$ & $136(134-140)$ & $137(135-138)$ \\
Serum potassium $(\mathrm{mmol} / \mathrm{L})$ & $4.0(4.0-4.3)$ & $4.1(3.8-4.4)$ \\
Serum creatinine $(\mu \mathrm{mol} / \mathrm{L})$ & $124(98-154)$ & $129(103-160)$ \\
Line-related complications & Not applicable & 0.462 \\
\hline
\end{tabular}


a shorter length of hospitalisation than the standard care group. This is likely due to the rapid volume removal that was achieved with UF, resulting in the quicker attainment of euvolaemia. We also showed that UF was associated with a significantly lower number of 90-day emergency department visits for HF, as well as a significantly lower number of 90-day rehospitalisations for HF. There was also a trend toward a numerically lower number of 30-day emergency department visits and rehospitalisations for HF in the UF group, although this did not reach statistical significance. The improved clinical outcomes observed with the use of UF may imply less resource utilisation for HF. In addition, the fact that UF can be performed safely in the general ward, as compared to the need for intensive care unit stay for standard care patients who require inotropic support, could represent potential healthcare cost savings, although this was not directly analysed in the present study. Further studies are needed to specifically address the impact of the use of UF on health economics.

The reductions in the number of emergency department visits and readmissions for HF suggest that UF results in sustained haemodynamic and neurohormonal benefits; this has also been demonstrated in previous studies. ${ }^{(22-24)}$ As compared to diuretics, UF has been shown to remove more total body sodium for similar volumes of fluid removed. ${ }^{(25)}$ The ultrafiltrate produced in UF is isotonic compared to plasma, whereas the urine produced by loop diuretics is hypotonic. ${ }^{(23,24)}$ Hence, UF has also been associated with decreased neurohormonal activation, as indicated by reduced levels of plasma brain natriuretic peptide, norepinephrine, renin and aldosterone, which do not occur with intravenous diuretics. ${ }^{(23)}$ In a study conducted by Marenzi et al, ${ }^{(22}$ the improved haemodynamics associated with the use of UF was reflected in reduced mean right atrial, pulmonary artery and wedge pressures, as well as increased cardiac output and stroke volume. ${ }^{(22)}$

The persistent clinical improvements following UF could also be explained by the restoration of diuretic responsiveness. ${ }^{(22,24)}$ It is widely known that prolonged diuretic use impairs the kidney's efficiency to produce urine in response to a certain amount of diuretic administered. It has been suggested that resistance to diuretics is an adverse prognostic indicator and that such resistance is associated with mortality in HF patients. ${ }^{(26-29)}$ Previous studies have demonstrated that HF patients who have undergone UF treatment require a lower dose of maintenance diuretics than patients who received standard care. ${ }^{(17,22)}$ In essence, by improving diuresis, natriuresis, haemodynamics and neurohormonal responses, UF is able to interrupt the vicious circle that leads to refractory HF.

Our experience with UF suggests that the relief in congestion that was achieved via UF could lead to a better quality of life. Improvement in the functional status of patients in the UF group was more pronounced than in those in the standard care group, as shown by the greater decline in EQ-5D scores of patients in the UF group at discharge and 30 days. This observation is consistent with that of other UF trials. ${ }^{(16,17,26)}$ Other than that, none of the patients who underwent UF in our study cohort experienced adverse events such as hypotension, bleeding, electrolyte imbalance, worsening renal function or line-related complications. This finding further confirms the safety of UF. ${ }^{15-17,26)}$

The efficacy and safety findings of UF in the present study are largely comparable to the proof-of-concept Relief for Acutely Fluid-Overloaded Patients with Decompensated Congestive Heart Failure (RAPID-CHF) trial and the landmark randomised UNLOAD trial. ${ }^{(16,17)}$ However, the latest randomised UF trial, Cardiorenal Rescue Study in Acute Decompensated Heart Failure (CARRESS-HF), failed to demonstrate the superiority of UF over pharmacologic therapy in their cardiorenal patient population. ${ }^{(30)}$ CARRESS-HF showed similar weight loss, mortality and rate of hospitalisation for HF between patients who received UF and patients who received diuretic treatment. One major difference between the study design of CARRESS-HF and the aforementioned studies (i.e. RAPID-CHF, UNLOAD and the present study) was that CARRESS-HF had a fixed UF rate of $200 \mathrm{~mL} / \mathrm{hr}$, while the other studies allowed a UF rate of up to $500 \mathrm{~mL} / \mathrm{hr}$. This could explain the lack of fluid and weight loss in the UF group in CARESS-HF. Furthermore, the renal function of the patients who underwent UF in CARRESS-HF worsened; the reason for this is unclear and has been postulated to be due to transient intravascular volume depletion during UF. ${ }^{(30)}$

It must be emphasised that the patient populations of each of these trials were different - the RAPID-CHF and UNLOAD trials recruited hypervolaemic HF patients, CARRESS-HF enrolled HF patients with cardiorenal syndrome, and the present study enrolled acutely decompensated HF patients with diuretic resistance. Our study cohort was selected in accordance with the guidelines for the use of UF by ACCF/AHA and ESC. This highlights the importance of selecting appropriate patients for UF. The results of our study demonstrated that UF can be used in acutely decompensated HF patients who are not responsive to diuretics. In this group of patients, UF was able to relieve congestion rapidly and safely, reduce the length of hospitalisation and the frequency of readmission due to HF, and improve the patient's quality of life. However, diuretics should still be the first-line treatment strategy for patients who are diuretic-sensitive, given the high cost and complexity of UF.

The principal limitation of the present study is that it is a single-centre, retrospective cohort study with a small study population. However, the outcome data of the study is generally in agreement with the two randomised UF trials, RAPID-CHF and UNLOAD. A second limitation is that there may have been inherent biases, as the use of inotropes and the doses of intravenous diuretics administered were at the discretion of the treating physician for the standard care group, while the rate and duration of UF were judiciously determined by the HF physician for the UF group. The treating physician of the standard care group might have been a cardiologist of other subspecialties, whereas the treating physician of the UF group was a HF specialist; this may represent a confounding factor and could have contributed to the different clinical outcomes between the two groups.

To conclude, our single-centre experience demonstrated that UF is an effective treatment for decompensated HF patients with diuretic resistance from an Asian population. UF can help such 
patients achieve greater fluid and weight loss, reduced length of hospitalisation, and lower frequency of emergency department visits and rehospitalisations. UF also improves the health status of such patients and can be performed safely without adverse events.

\section{REFERENCES}

1. Go AS, Mozaffarian D, Roger VL, et al; American Heart Association Statistics Committee and Stroke Statistics Subcommittee. Heart disease and stroke statistics--2014 update: a report from the American Heart Association. Circulation 2014; 129:e28-e292.

2. McMurray JJ, Petrie MC, Murdoch DR, Davie AP. Clinical epidemiology of heart failure: public and private health burden. Eur Heart J 1998; 19 Suppl P: P9-16.

3. Ng TP, Niti M. Trends and ethnic differences in hospital admissions and mortality for congestive heart failure in the elderly in Singapore, 1991 to 1998. Heart 2003; 89:865-70.

4. Leong KT, Goh PP, Chang BC, Lingamanaicker J. Heart failure cohort in Singapore with defined criteria: clinical characteristics and prognosis in a multi-ethnic hospital-based cohort in Singapore. Singapore Med J 2007; 48:408-14.

5. Yancy CW, Jessup M, Bozkurt B, et al; American College of Cardiology Foundation, American Heart Association Task Force on Practice Guidelines. 2013 ACCF/AHA guideline for the management of heart failure: a report of the American College of Cardiology Foundation/American Heart Association Task Force on Practice Guidelines. J Am Coll Cardiol 2013; 62:e147-239.

6. Adams KF Jr, Fonarow GC, Emerman CL, et al; ADHERE Scientific Advisory Committee and Investigators. Characteristics and outcomes of patients hospitalized for heart failure in the United States: rationale, design, and preliminary observations from the first 100,000 cases in the Acute Decompensated Heart Failure National Registry (ADHERE). Am Heart J 2005; 149:209-16.

7. Krumholz HM, Chen YT, Vaccarino V, et al. Correlates and impact on outcomes of worsening renal function in patients $>$ or $=65$ years of age with heart failure. Am J Cardiol 2000; 85:1110-3.

8. Butler J, Forman DE, Abraham WT, et al. Relationship between heart failure treatment and development of worsening renal function among hospitalized patients. Am Heart J 2004; 147:331-8.

9. Valente MA, Voors AA, Damman K, et al. Diuretic response in acute heart failure: clinical characteristics and prognostic significance. Eur Heart J 2014; 35:1284-93.

10. De Bruyne LK. Mechanisms and management of diuretic resistance in congestive heart failure. Postgrad Med J 2003; 79:268-71.

11. Ellison DH. Diuretic therapy and resistance in congestive heart failure. Cardiology 2001; 96:132-43.

12. Leto L, Aspromonte N, Feola M. Efficacy and safety of loop diuretic therapy in acute decompensated heart failure: a clinical review. Heart Fail Rev 2014; 19:237-46.

13. Kolff WJ, Leonards JR. Reduction of otherwise intractable edema by dialysis or filtration. Cleve Clin Q 1954; 21:61-71.

14. Simpson IA, Rae AP, Simpson K, et al. Ultrafiltration in the management of refractory congestive heart failure. Br Heart J 1986; 55:344-7.

15. Donato L, Biagini A, Contini C, et al. Treatment of end-stage congestive heart failure by extracorporeal ultrafiltration. Am J Cardiol 1987; 59:379-80.

16. Jaski BE, Ha J, Denys BG, et al. Peripherally inserted veno-venous ultrafiltration for rapid treatment of volume overloaded patients. J Card Fail 2003; 9:227-31.

17. Bart BA, Boyle A, Bank AJ, et al. Ultrafiltration versus usual care for hospitalized patients with heart failure: the Relief for Acutely FluidOverloaded Patients with Decompensated Congestive Heart Failure (RAPID-CHF) trial. J Am Coll Cardiol 2005; 46:2043-6.

18. Costanzo MR, Guglin ME, Saltzberg MT, et al; UNLOAD Trial Investigators. Ultrafiltration versus intravenous diuretics for patients hospitalized for acute decompensated heart failure. J Am Coll Cardiol 2007; 49:675-83.

19. EQ-5D Nomenclature. In: EuroQol [online]. Available at: http://www. euroqol.org/about-eq-5d/eq-5d-nomenclature.html. Accessed August 11, 2011.

20. McMurray JJ, Adamopoulos S, Anker SD, et al; ESC Committee for Practice Guidelines. ESC Guidelines for the diagnosis and treatment of acute and chronic heart failure 2012: The Task Force for the Diagnosis and Treatment of Acute and Chronic Heart Failure 2012 of the European Society of Cardiology. Developed in collaboration with the Heart Failure Association (HFA) of the ESC. Eur Heart J 2012; 33:1787-847.

21. Patarroyo M, Wehbe $E$, Hanna $M$, et al. Cardiorenal outcomes after slow continuous ultrafiltration therapy in refractory patients with advanced decompensated heart failure. J Am Coll Cardiol 2012; 60:1906-12.

22. Marenzi G, Lauri G, Grazi M, et al. Circulatory response to fluid overload removal by extracorporeal ultrafiltration in refractory congestive heart failure. J Am Coll Cardiol 2001; 38:963-8

23. Agostoni P, Marenzi G, Lauri G, et al. Sustained improvement in functional capacity after removal of body fluid with isolated ultrafiltration in chronic cardiac insufficiency: failure of furosemide to provide the same result. Am Jed 1994; 96:191-9.

24. Guazzi MD, Agostoni P, Perego B, et al. Apparent paradox of neurohumoral axis inhibition after body fluid volume depletion in patients with chronic congestive heart failure and water retention. Br Heart J 1994; 72:534-9.

25. Schrier RW. Role of diminished renal function in cardiovascular mortality: marker or pathogenetic factor? J Am Coll Cardiol 2006; 47:1-8.

26. Costanzo MR, Saltzberg M, O'Sullivan J, Sobotka P. Early ultrafiltration in patients with decompensated heart failure and diuretic resistance. J Am Coll Cardiol 2005; 46:2047-51.

27. Felker GM, Mentz RJ. Diuretics and ultrafiltration in acute decompensated heart failure. J Am Coll Cardiol 2012; 59:2145-53.

28. Neuberg GW, Miller AB, O'Connor CM, et al; PRAISE Investigators, Prospective Randomized Amlodipine Survival Evaluation. Diuretic resistance predicts mortality in patients with advanced heart failure. Am Heart J 2002; 144:31-8.

29. Eshaghian S, Horwich TB, Fonarow GC. Relation of loop diuretic dose to mortality in advanced heart failure. Am J Cardiol 2006; 97:1759-64.

30. Bart BA, Goldsmith SR, Lee KL, et al; Heart Failure Clinical Research Network. Ultrafiltration in decompensated heart failure with cardiorenal syndrome. N Engl J Med 2012; 367:2296-304. 